\title{
Static and dynamic effects of the incomplete ionization in SuperJunction devices
}

\author{
Nazareno Donato and Florin Udrea, Member, IEEE
}

\begin{abstract}
The incomplete ionization of impurity atoms affects the free carrier concentration of several wide bandgap semiconductor materials even at room temperature, thus modifying the electrical properties of power devices. In this paper, the influence of the partial ionization of the dopants on the static and dynamic behavior of wide bandgap semiconductor based SuperJunction devices has been numerically investigated through extensive 2D finite element simulations. Whereas this physical effect has only a minor impact on the static device' characteristics, if a reverse bias pulse with a rise time comparable or smaller than the ionization time constant is applied to the structure, a "dynamic ionization" phenomenon can take place. The onset of this time dependent ionization is the cause of charge unbalance effects in the device structure, due to temporal dependence of the activated number of dopants. Electro-thermal simulations, which have been carried out for a $4 \mathrm{H}-\mathrm{SiC}$ SuperJunction diode, show a nonuniform temperature distribution during the transients which leads, in turn, to the self-heating of the device. Through an accurate redesign of the cross-sectional view of the device, the drawbacks of the incomplete ionization have been mitigated and the device' performance enhanced.
\end{abstract}

Index Terms - incomplete ionization, modelling, power devices, Silicon Carbide, SuperJunction, TCAD.

\section{INTRODUCTION}

$\mathrm{T}$ he invention of the SuperJunction (SJ) concept $[1,2]$ and the advent of wide bandgap (WBG) semiconductors [3-5] have provided new opportunities to break the Silicon limit[6-8] and enhance the performance of power semiconductor devices. On one hand, the SJ consists in the replacement of the drift region with multiple uniformly doped p-type and n-type columns $[6,9,10]$. In the case of unipolar devices, this allows for a much higher doping concentrations of the pillars for a fixed breakdown voltage (when the charge compensation is maintained between the different pillars), and, for this specific reason, improves the trade-off between specific on-state resistance $\left(\mathrm{R}_{\text {on } \_ \text {spec }}\right)$ and the breakdown voltage $(\mathrm{BV})[6]$. On the other hand, the superior material properties, such as the higher critical electric field, the higher saturation velocity, thermal conductivity, etc. of WBG semiconductors (Silicon Carbide, Gallium Nitride, Diamond) compared to Silicon (Si), allow for reduced device size and faster switching frequencies, for the same BV[11]. This, in turn, has also a positive impact on the $\mathrm{R}_{\text {on_spec, }}$, which is lowered by the significant reduction of the drift region thickness. In the past, several research groups have tried to combine the benefits of SJ and WBG semiconductors. In the case of Gallium Nitride (GaN), the SJ concept has mainly resulted in the "natural SuperJunction" idea [12, 13], in which the depletion of the alternate $2 \mathrm{DEG}$ and $2 \mathrm{DHG}$ generates a charge compensation effect similar to the classical Si SJ theory. In Silicon Carbide ( $\mathrm{SiC}$ ), the $\mathrm{SJ}$ idea has been demonstrated in Schottky diodes $[14,15]$ and recently in a V-Groove Trench MOSFET structure[16]. In Diamond (C), the restrictions due to the low efficiency and control of p-type and especially n-type doping[17], have hindered, so far, the fabrication of diamond SJ devices. One significant drawback of WBG semiconductors is the incomplete ionization [18], a physical effect which occurs in great part of the dopant species (table 1). In this scenario, the modelling of the electro-thermal device characteristics becomes a fundamental tool for the prediction of real device performance and any subsequent optimization. The possible consequences of the deep level nature of acceptors and donors on the design of SJ power devices have not been investigated yet. In this paper, the static and dynamic electro-thermal effects of the incomplete ionization have been deeply studied for the case of a $4 \mathrm{H}-\mathrm{SiC} \mathrm{SJ}$ diode. In detail, the effects caused by the dynamic ionization of the impurities in the two pillars of the SJ diode have been examined by means of electro-thermal simulations carried out with Sentaurus Technology Computer-Aided Design (TCAD) [19].

\section{A. Theory of the incomplete ionization}

TABLE 1

ACTIVATION ENERGIES FOR DIFFERENT DOPANT ATOMS IN WBG SEMICONDUCTORS.

\begin{tabular}{|l|l|c|}
\hline Material & Dopant species & Activation energy \\
\hline $4 H-S i C$ & B (p-type) & $0.293 \mathrm{eV}$ \\
& Al (p-type) & $0.265 \mathrm{eV}$ \\
& N (n-type) & $0.07 \mathrm{eV}(\mathrm{Hex}) \quad 0.12 \mathrm{eV}(\mathrm{K})$ \\
& $\mathrm{P}$ (n-type) & $0.055 \mathrm{eV}(\mathrm{Hex}) \quad 0.102 \mathrm{eV}(\mathrm{K})$ \\
\hline GaN & Mg (p-type) & $0.16 \mathrm{eV}$ \\
& C (p-type) & $0.9 \mathrm{eV}$ \\
\hline Diamond & B (p-type) & $0.37 \mathrm{eV}$ \\
& P (n-type) & $0.57 \mathrm{eV}$ \\
& N (n-type) & $1.7 \mathrm{eV}$ \\
\hline
\end{tabular}

The incomplete ionization of the dopants has a profound impact on the electrical performance of WBG devices. In case of 4H$\mathrm{SiC}$, it is worth noting that different activation energies may arise from different positions in the lattice crystal, i.e. hexagonal (Hex) or cubic (K) sites (table 1). The modelling of 
the incomplete ionization phenomenon can be done by conceiving the dopant species as acceptors and donors impurities coupled with the valence and conductance band, respectively, within the framework of the electrothermal driftdiffusion model[20]. The presence of deep level impurities can be treated with the Fermi-Dirac distribution, also known as steady-state Gibbs distribution, as illustrated in (1a) and (1b).

$$
N_{D}=\frac{N_{D 0}}{1+g_{D} \cdot \exp \left(\frac{E_{F N}-E_{D}}{k T}\right)} \quad \text { (1a) } \quad N_{A}=\frac{N_{A 0}}{1+g_{A} \cdot \exp \left(\frac{E_{A}-E_{F P}}{k T}\right)}
$$

Where $E_{\mathrm{FN}}\left(\mathrm{E}_{\mathrm{FP}}\right)$ is the quasi-Fermi energy level for electrons (holes), $g_{D}\left(g_{A}\right)$ is the degeneracy factor for donors (acceptors), $\mathrm{N}_{\mathrm{D}}\left(\mathrm{N}_{\mathrm{A}}\right)$ is the activated number of donors (acceptors), $\mathrm{N}_{\mathrm{D} 0}$ $\left(\mathrm{N}_{\mathrm{A} 0}\right)$ is the total number of donors (acceptors), $\mathrm{E}_{\mathrm{D}}\left(\mathrm{E}_{\mathrm{A}}\right)$ is the energy level for donors (acceptors), $\mathrm{T}$ is the absolute temperature in Kelvin and $\mathrm{k}$ the Boltzmann constant. In table 1, the activation energy is defined as $E_{C}-E_{D}$ for donors and $E_{A}-E_{V}$ for acceptors, where $E_{C}$ and $E_{V}$ are the minimum and the maximum energy level of the conductance and valance band, respectively. When a certain critical acceptor dopant concentration $\mathrm{N}_{\text {Acritic }}$ (or $\mathrm{N}_{\text {Dcritic }}$ for donors) is reached, full activation can be considered (i.e. $\mathrm{N}_{\mathrm{A}}=\mathrm{N}_{\mathrm{A} 0}$ for $\mathrm{N}_{\mathrm{A} 0} \geq \mathrm{N}_{\mathrm{Acritic}}$ and $\mathrm{N}_{\mathrm{D}}=\mathrm{N}_{\mathrm{D} 0}$ when $\mathrm{N}_{\mathrm{D} 0} \geq \mathrm{N}_{\text {Drritic }}$. In the formulas (1), the variation of the activation energy due to the Poole-Frenkel effect has been neglected whilst the Pearson and Bardeen formula which accounts for a reduction of the activation energy at high doping concentration has been considered, as in [21]. The equilibrium concentrations in a semiconductor bulk region can be evaluated by finding the Fermi energy level $\mathrm{E}_{\mathrm{F}}\left(\mathrm{E}_{\mathrm{FN}}=\mathrm{E}_{\mathrm{FP}}=\mathrm{E}_{\mathrm{F}}\right.$ at the equilibrium) which simultaneously satisfies the charge neutrality condition $\left(n+N_{A}=p+N_{D}\right)$ for electrons (n) and holes $(p)$ and the equations ( $1 \mathrm{a})$ and (1b). This merging, together with the law of mass action $\left(p n=n_{i}^{2}\right)$, leads to the formula (2a) and (2b), for n-type and p-type bulk regions respectively.

$\frac{n\left(n+N_{A 0}\right)-n_{i}^{2}}{N_{D 0}-N_{A 0}-n+\frac{n_{i}^{2}}{n}}=\frac{N_{C}}{g_{D}} \cdot \exp \left(-\frac{E_{C}-E_{D}}{k T}\right)$

$\frac{p\left(p+N_{D 0}\right)-n_{i}^{2}}{N_{A 0}-N_{D 0}-p+\frac{n_{i}^{2}}{p}}=\frac{N_{V}}{g_{A}} \cdot \exp \left(-\frac{E_{A}-E_{V}}{k T}\right)$

Where $\mathrm{n}_{\mathrm{i}}$ intrinsic carrier concentration (in $\left.\mathrm{cm}^{-3}\right), \mathrm{N}_{\mathrm{V}}\left(\mathrm{N}_{\mathrm{C}}\right)$ the density of states in the valence (conductance) band and $\mathrm{N}_{\mathrm{A} 0}$ $\left(\mathrm{N}_{\mathrm{D} 0}\right)$ in formula $2 \mathrm{a}(2 \mathrm{~b})$ indicates the amount of compensation doping which has been considered as fully activated (i.e. $\mathrm{N}_{\mathrm{A}}=\mathrm{N}_{\mathrm{A} 0}$ for 2(a) and $\mathrm{N}_{\mathrm{D}}=\mathrm{N}_{\mathrm{D} 0}$ for 2(b)). It is worth noting that the parameters $\mathrm{n}_{\mathrm{i}}, \mathrm{N}_{\mathrm{V}}, \mathrm{N}_{\mathrm{C}}, \mathrm{g}_{\mathrm{A}}, \mathrm{g}_{\mathrm{D}}$ are assumed to be temperature dependent[22]. In the non-neutral regions (such as depletion regions), where the electrostatic potential $\Psi$ is not negligible and in general given by the Poisson equation, the charge neutrality condition does not hold anymore, and the concentration can be evaluated as in (3a) and (3b).

$\begin{aligned} N_{D} & =\frac{N_{D 0}}{1+g_{D} \cdot \exp \left(\frac{E_{F N}-E_{D}}{k T}\right) \cdot \exp \left(\frac{q \Psi}{k T}\right)} \\ N_{A} & =\frac{N_{A 0}}{1+g_{A} \cdot \exp \left(\frac{E_{A}-E_{F P}}{k T}\right) \cdot \exp \left(-\frac{q \Psi}{k T}\right)}\end{aligned}$

Where $\mathrm{E}_{\mathrm{FN}}, \mathrm{E}_{\mathrm{FP}}, \mathrm{E}_{\mathrm{A}}$ and $\mathrm{E}_{\mathrm{D}}$ are the energy levels in the neutral region, which coincide with the ones calculated at zero bias and under the charge neutrality condition. With reference to a classic pn junction, in the depletion region the electrostatic potential $\Psi$ is positive in the p-side (negative in the n-side) with respect to the p-type (n-type) bulk[20]. When a reverse voltage is applied to the junction, both the potential barrier and the width of the depletion regions increase. For this reason, the denominator of (3a) and (3b) approaches the unity and $\mathrm{N}_{\mathrm{D}} \sim \mathrm{N}_{\mathrm{D} 0}$ such as $\mathrm{N}_{\mathrm{A}} \sim \mathrm{N}_{\mathrm{A} 0}$ in the $\mathrm{n}$-side and $\mathrm{p}$-side, respectively, so causing a full activation of the dopant species. Whereas the electrostatic of the incomplete ionization is mainly governed by equations (1) and (3), the dynamic time response is highly dependent on the capture and emission rates, as shown in equations (4a-d)[23]. In these equations, $t$ is the time, $v_{\text {th }} \mathrm{e}\left(\mathrm{v}_{\mathrm{th} \_} \mathrm{h}\right)$ are the electron (hole) thermal velocity, $\sigma_{\mathrm{D}}\left(\sigma_{\mathrm{A}}\right)$ is the cross section for donors (acceptors) species.

$$
\begin{aligned}
& \frac{\partial N_{D}}{\partial t}=v_{t h \_} \cdot \sigma_{D}\left[\frac{n_{1}}{g_{D}} N_{D 0}-\left(n+\frac{n_{1}}{g_{D}}\right) N_{D}\right](4 \mathrm{a}) \quad n_{1}=N_{C} \cdot \exp \left(-\frac{E_{C}-E_{D}}{k T}\right)(4 \mathrm{~b}) \\
& \frac{\partial N_{A}}{\partial t}=v_{t h_{-} h} \cdot \sigma_{A}\left[\frac{p_{1}}{g_{A}} N_{A 0}-\left(p+\frac{p_{1}}{g_{A}}\right) N_{A}\right] \quad(4 \mathrm{c}) \quad p_{1}=N_{V} \cdot \exp \left(-\frac{E_{A}-E_{V}}{k T}\right)(4 \mathrm{~d})
\end{aligned}
$$

The main idea behind the dynamic model of the incomplete ionization is that acceptor and donor dopant atoms can be viewed as donor and acceptor traps respectively. Indeed, the carriers' dynamic shown in equations (4a-d) is a generalization of the Shockley-Read-Hall (SRH) model in case of transient conditions[24]. It is possible to rewrite the equation (4c) and then define a time constant for the incomplete ionization model, as illustrated in (5) in case of acceptor dopants. This time constant $\left(\tau_{\mathrm{p}}\right)$ is highly dependent on the capture and emission rates defined in (6) and for this reason, it depends on the activation energy, temperature, cross section etc. The same derivations can be done for donor impurities.

$$
\begin{aligned}
& \frac{\partial N_{A}}{\partial t}=-\left(e_{p}+c_{p} p\right) N_{A}+e_{p} N_{A 0} \quad \text { (5a) } \quad \tau_{p}=\frac{1}{e_{p}+c_{p} p} \\
& c_{p}=v_{t h_{-} h} \cdot \sigma_{A} \\
& e_{p}=v_{t h_{-} h} \cdot \sigma_{A} \cdot N_{V} \cdot g_{A}^{-1} \cdot \exp \left(-\frac{E_{A}-E_{V}}{k T}\right)
\end{aligned}
$$

If the device under test (DUT) is subjected to a dynamic process (i.e. $\mathrm{dV} / \mathrm{dt}$ or $\mathrm{dI} / \mathrm{dt}$ ) with a time constant smaller than the ionization/deionization, the static equations in (1) do not hold anymore and equations (4) have to be solved. The evolution of the carrier activation with time is then governed by the incomplete ionization time constant. Inside the space charge region ( $\mathrm{SCR})$, the time constant defined in $(5 \mathrm{~b})$ modifies into (7) as no free carriers are present in the structure.

$\tau_{p}=\frac{1}{e_{p}}$

Under this specific condition, the time constant does not depend upon the capture rate and it can be easily evaluated as shown in figure 1 for boron doped $4 \mathrm{H}-\mathrm{SiC}$.

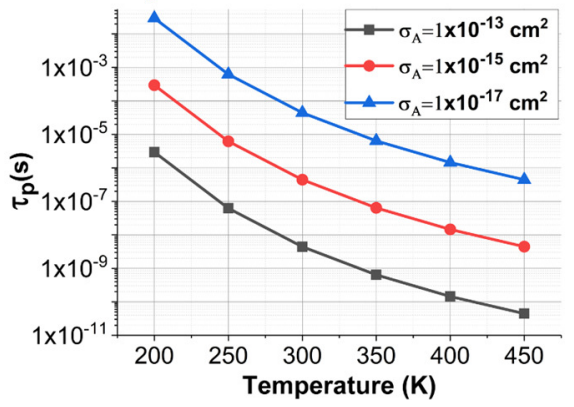

Figure 1, Ionization time constant vs temperature for three different values of the cross section for a $4 \mathrm{H}-\mathrm{SiC}$ boron doped layer. In equation (6b), $\mathrm{N}_{\mathrm{V}}$ and $\mathrm{v}_{\text {th }} \mathrm{h}$ are assumed temperature dependent. 
It is worth noting that the cross section can be also electric field and temperature dependent. In this paper, we considered a constant value of $\sigma_{\mathrm{A}}\left(1 \times 10^{-15} \mathrm{~cm}^{2}\right)$ for boron and nitrogen doped $4 \mathrm{H}-\mathrm{SiC}$, a mean value according to several DLTS measurements reported in $[25,26]$. Due to the very low value of the activation energy and the doping values adopted in this study, both the dynamic and static effects of the incomplete ionization on nitrogen doped $4 \mathrm{H}-\mathrm{SiC}$ could be neglected. The effects of the incomplete ionization on the modelling of a $4 \mathrm{H}-$ $\mathrm{SiC}$ SJ diode will be discussed in the next section.

\section{4H-SIC SUPERJUNCTION DIODE}

\section{A. On-state and static off-state}

(a)

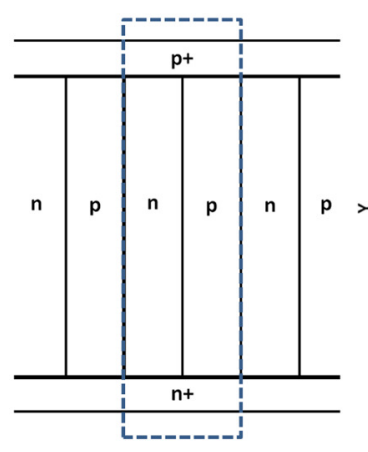

(b)

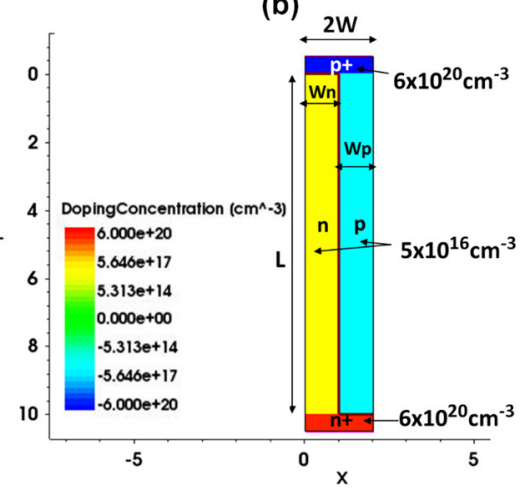

Figure 2, cross section of 4H-SiC SJ diode structure (a) and unit cell adopted for the TCAD simulations(b). The dopant concentration of the p-type and ntype pillar $\left(\mathrm{N}_{\mathrm{A} 0}, \mathrm{~N}_{\mathrm{D} 0}\right.$ respectively) has been fixed at $5 \times 10^{16} \mathrm{~cm}^{-3}$ for both pillars, whilst the concentration of the $\mathrm{n}+$ and $\mathrm{p}+$ regions is set at $6 \times 10^{20} \mathrm{~cm}^{-3}$. The aspect ratio $(\mathrm{L} / \mathrm{W})$ of the structure is $10(\mathrm{~L}=10 \mu \mathrm{m}$ and $\mathrm{W}=\mathrm{Wp}=\mathrm{Wn}=1 \mu \mathrm{m})$.

The cross-sectional view for the unit cell of the $4 \mathrm{H}-\mathrm{SiC} \mathrm{SJ}$ diode is shown in figure 2. The critical value of the dopant concentration after which the total activation occurs has been fixed at $1 \times 10^{22} \mathrm{~cm}^{-3}$. The values for the other parameters adopted for the TCAD simulations (mobility, impact ionization, bandgap etc.) can be found in default $4 \mathrm{H}-\mathrm{SiC}$ parameter file [19]. The dopant species are nitrogen $(\mathrm{N})$ for the n-type layers (Hex site) and boron (B) for the p-type layers. B has been chosen as p-type dopant instead of $\mathrm{Al}$ for the TCAD simulations, but all the considerations done for this dopant species may be applied also for the case of Al dopant or for other WBG semiconductors SJ devices, in which the incomplete ionization' effect cannot be neglected (i.e. Diamond, $\mathrm{GaN}, \mathrm{\beta Ga}_{2} \mathrm{O}_{3}$, etc). If one assumes that $\mathrm{Wp}=\mathrm{Wn}=\mathrm{W}$ and $\mathrm{N}_{\mathrm{A} 0}=\mathrm{N}_{\mathrm{D} 0}$ (as in figure 2(b)), and then solves (2a) and (2b) to evaluate the activated number of acceptor $\left(\mathrm{N}_{\mathrm{A}}\right)$ and donor dopants $\left(\mathrm{N}_{\mathrm{D}}\right)$, the theoretical unbalance ratio between the n-type pillar and the p-type pillar at different temperature can be easily calculated, as shown in figure 3(a). An unbalance ratio between the SJ pillars, would lead to a drastic reduction of the breakdown capability of the device, a well-known effect in the classic SJ theory[27], as also illustrated in figure 3(b). However, if the SJ diode is properly designed, a full lateral and vertical depletion of the device' structure occurs before the BV (which is due to the avalanche generation under our assumptions). In particular, if the electric field is high enough in all the structure (i.e. the device is all depleted), it may be able to activate all the dopants(i.e. $\mathrm{N}_{\mathrm{A}} \sim \mathrm{N}_{\mathrm{A} 0}$ and $\mathrm{N}_{\mathrm{D}} \sim \mathrm{N}_{\mathrm{D} 0}$ ), as already discussed in the previous paragraph.
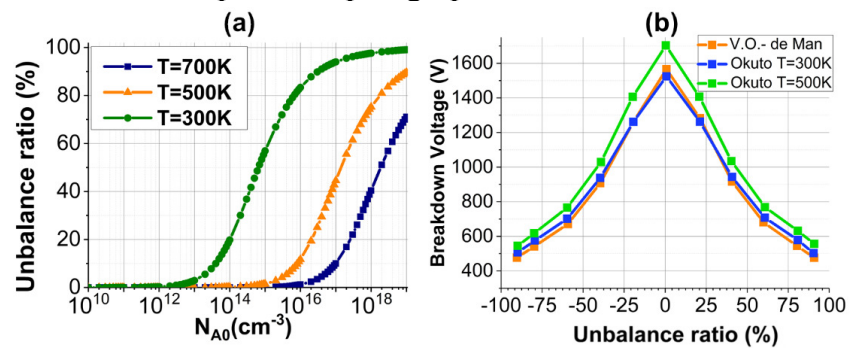

Figure 3, (a) (Theoretical) Unbalance ratio vs dopant concentration $\left(\mathrm{N}_{\mathrm{A} 0}\right.$, which is assumed equal to $\mathrm{N}_{\mathrm{D} 0}$ ) for three different operating temperatures of the device depicted in figure 2. The unbalance ratio for the device structure in figure 2(b) has been defined as $100 * \operatorname{abs}\left(\mathrm{N}_{\mathrm{A}}-\mathrm{N}_{\mathrm{D}}\right) / \max \left(\mathrm{N}_{\mathrm{A}}, \mathrm{N}_{\mathrm{D}}\right)$. (b) Simulated $\mathrm{BV}$ vs Unbalance ratio for the device structure shown in figure 2 with two different impact ionization models (Van Overstraeten-de Man and Okuto[19, 28, 29]). Okuto model, which takes into account a positive temperature coefficient of the breakdown voltage, has been selected as the avalanche model for this study.

Indeed, if one balances the SJ structure assuming total ionization in both pillars while satisfying the charge balance condition (as in device shown in figure 2(b)), the breakdown voltage is identical with and without the incomplete ionization model, meaning that the electric field is actually able to activate virtually all of the dopants during the off-state. This means that the static BV for the device depicted in figure 2(b) corresponds to the value shown in figure 3(b) for an unbalance ratio of $0 \%$. Regarding the on-state (figure 4), if the conductive pillar is the one affected by the incomplete ionization, the current density is highly reduced, and the risk may be a total depletion of the ptype pillar with consequential no current flowing in the device. The device adopted for the on-state simulation is shown in figure 4(a) where a top $\mathrm{p}^{+}$layer has been introduced for structure \#1B in order to emulate the flow of the majority carriers which occurs in a real MOSFET structure. It is also evident that the high field dependence model[30] which accounts for a reduction of the mobility for high electric field values, also plays a key role in the on-state, emphasizing the JFET effect[31]. (a)

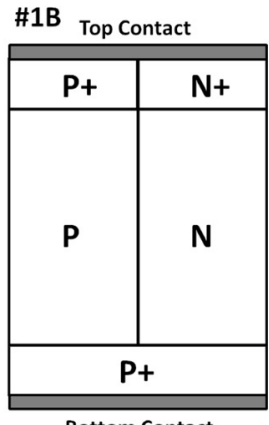

Bottom Contact (b)

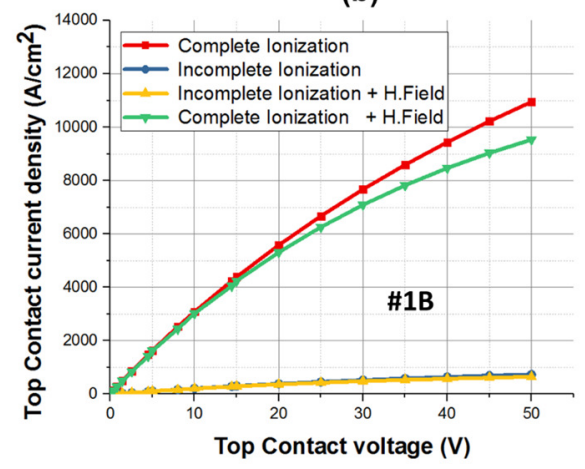

Figure 4, cross section of the device structure \#1B adopted for the on-state simulation (a) and on-state current density simulations (b).

\section{B. The dynamic unbalance}

As already discussed in the theory section, the ionization/deionization process is not only temperature but also time dependent. So, if a reverse bias pulse is applied to the structure and the rise time of this pulse is lower or comparable with the ionization time constant, not all the dopants are 
activated by the high electric field [18, 23]. In the specific case of a the $4 \mathrm{H}-\mathrm{SiC} \mathrm{SJ}$ diode, this dynamic ionization effect may lead to a charge unbalance situation (such as the one predicted in figure 3(a)) and reduce the real breakdown capability of the SJ. In order to investigate the possible consequences of the dynamic ionization, a reverse pulse of $1 \mathrm{kV} / 10 \mathrm{~ns}$ has been applied to the structure (figure 5). The rise time has been chosen taking into account the ionization time constant plotted in figure 1 in order to trigger the dynamic of the incomplete ionization. In practice, in devices such as MOSFETs, such high $\mathrm{dV} / \mathrm{dt}$ are unacceptable due to other effects such as system oscillations or retriggering of on-state conduction via the Miller capacitance and gate resistance[32]. However, this value is comparable with the maximum $\mathrm{dV} / \mathrm{dt}$ ruggedness reported in several datasheets of commercially available $\mathrm{SiC}$ devices[33, 34]. It is worth mentioning that the voltage applied to the SJ diode in this simulation is much lower than the static breakdown voltage (figure 3(b)) obtained in case of perfect balance (around $1.5 \mathrm{kV}$ at RT). The simulation results of the current density vs time clearly show that if the incomplete ionization is included in the simulation set, the SJ diode operates in avalanche condition for the whole duration of the pulse. The simulated current density is comparable with the on-state current density (figure 4). When the voltage pulse is removed, the SJ slightly recovers to its initial balanced condition and the current is mainly capacitive. To confirm the avalanche operation and the loss of the charge balance in reverse condition, the electric field has been plotted at the end of the rise time of the pulse $\left(\mathrm{V}=1 \mathrm{kV}\right.$ and $\left.\mathrm{t}=2.1 \times 10^{-7} \mathrm{~s}\right)$ in figure 6.

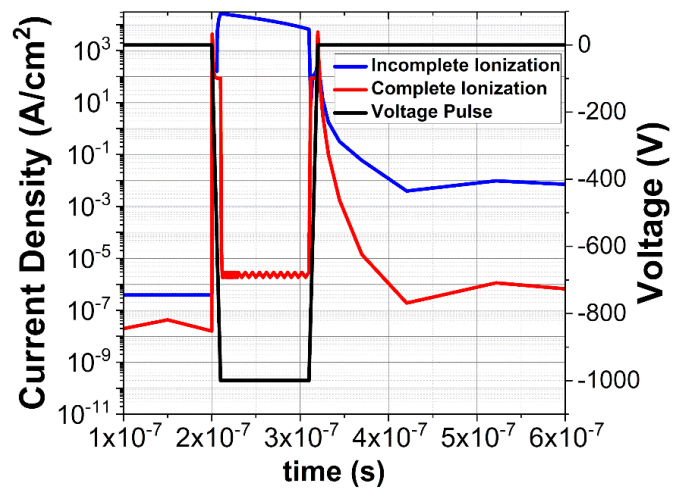

Figure 5, current density vs time with and without the incomplete ionization model. The rise time and the fall time of the reverse pulse are fixed at $10 \mathrm{~ns}$ $(\mathrm{tr}=\mathrm{tf}=10 \mathrm{~ns})$ and the duration of the pulse is set at $100 \mathrm{~ns}$ (ton=100ns).

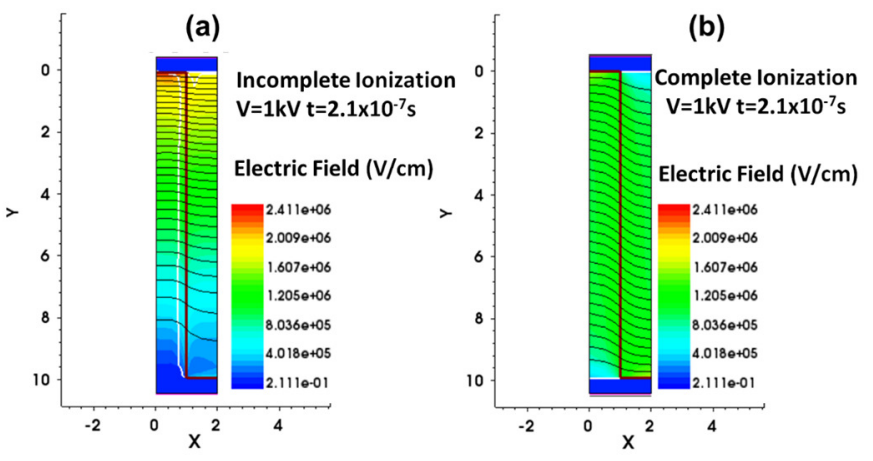

Figure 6, electric field and electrostatic potential contour at the end of the rise time of the pulse with (a) and without (b) incomplete ionization. With the incomplete ionization model, a clear unbalance can be deduced from both the non-uniform electrostatic potential contour and the higher electric field.
As the time passes the boron dopant activation increases and therefore the unbalance is dynamically lowered. This is also confirmed by the current density decrease over the time (constant slope of the blue curve in figure 5).

\section{Temperature effects: single and multiple pulse}

In the previous set of simulations, the Poisson and the current' equations have been computed without simultaneously solving the temperature equations at runtime. This assumption does not allow for investigating self-heating effects in the device which is operating under avalanche condition for the whole duration of the reverse pulse (figure 5) and is therefore subjected to high current density and applied voltage. In order to investigate the possible electro-thermal effects, two thermal resistances of a fixed value have been connected at the anode and cathode of the DUT, as schematically shown in figure 7.

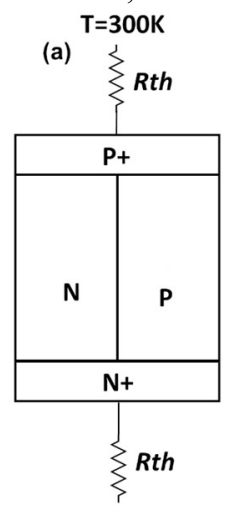

(b)

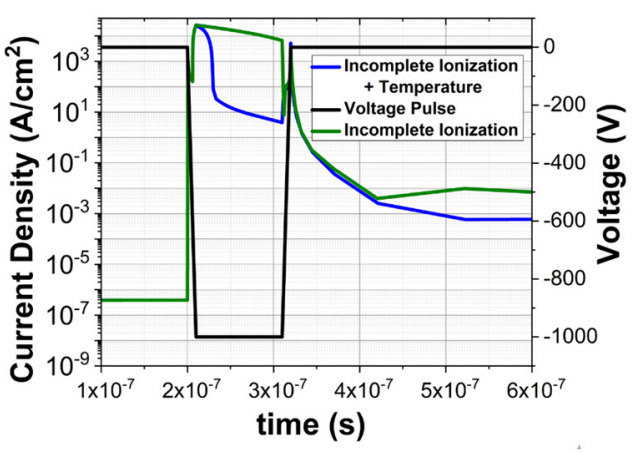

Figure 7, (a)schematic unit cell structure adopted for the TCAD electro-thermal simulations. A fixed value of the thermal resistance has been chosen for the top and bottom contact $(0.1 \mathrm{~K} / \mathrm{W})$. (b) current density vs time with and without the thermodynamic model. The specifications of the pulse are identical to figure 5 .

The thermodynamic model $[19,35]$ which allows to compute the temperature equations in each mesh point, has been adopted for the simulations shown in this work. In figure 7 , the simulation results of the fast reverse pulse applied to the SJ have been compared with and without the thermodynamic model, whilst the lattice temperature has been plotted in figure 8 .
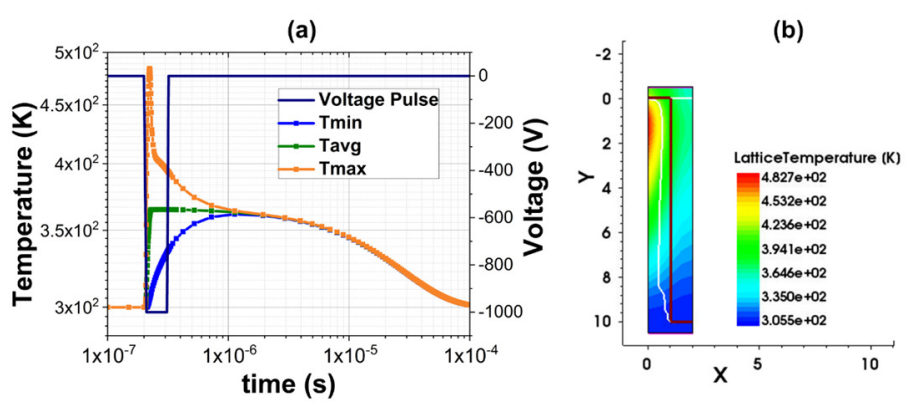

Figure 8 , temperature evolution in the structure as function of the time(a). The maximum temperature (Tmax) is observed at $t \sim 2.24 \times 10^{-7} \mathrm{~s}$ close to the maximum electric field point (b). Tmax slowly converges to the minimum (Tmin) and the average temperature (Tavg) for $\mathrm{t} \geq 1$ us. The device reaches RT only after $0.1 \mathrm{~ms}$.

The current density observed with the thermodynamic model dynamically reduces much faster than the one computed with the simple Poisson equation and the standard drift-diffusion model. Regarding the temperature, the maximum temperature is obtained at $\mathrm{t} \sim 2.24 \times 10^{-7} \mathrm{~s}$ and it is equal to $482 \mathrm{~K}$. This maximum temperature is observed, as expected, near the 
electric field peak point (figure 8). While the temperature rises due to the high power density $(\mathrm{P}=\mathrm{IV})$, the carrier activation increases much faster as it can be deduced from equations (1), the ionization time constant reduces exponentially as it can be observed from equations (7) and figure 1, and the avalanche generation is also reduced (avalanche is an electro-thermal stable phenomenon in the Okuto model). All these 3 effects combined, lead to an overall decrease of the transient current density as the balance is restored much faster. In figure 9, the carrier activation is compared with and without the thermodynamic model. The evolution of the temperature is observed also in case of a double pulse applied to the structure. As it can be observed from figure 10, the unbalance effects observed during the second pulse are much smaller than the one during the first pulse. Indeed, during the second $\mathrm{dV} / \mathrm{dt}$, the ionization time constant is smaller than during the first pulse because of the increased average temperature which also improves the breakdown voltage and the carrier activation in the structure, as previously discussed.
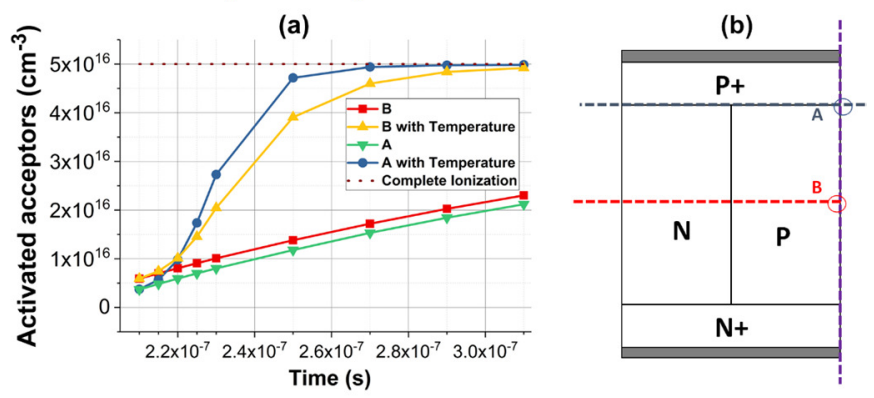

Figure 9, activated acceptors as function of time in two different points of the device structure with and without the thermodynamic model. The slope of the carrier activation vs time loses its linearity with the thermodynamic model. (a)

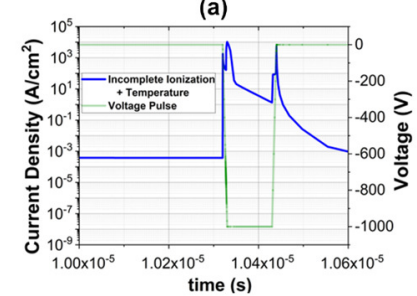

(b)

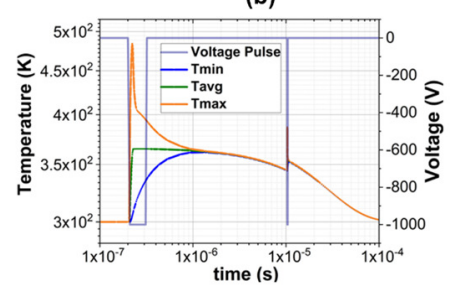

Figure 10, (a) transient evolution of the current density during the second pulse. The specifications of each pulse are the same of the single pulse (tr,tf,ton) and the dead time (tdead) between the two pulses has been chosen to be $10 \mu \mathrm{s}$. (b) Temperature observed in the case of a double pulse applied to the SJ diode. The application of a second pulse does not play a significant role in increasing the maximum temperature of the device which has a smaller peak (380K) compared to the one observed during the first transient (482K).

If multiple pulses are applied to the structure the same conclusions can be drawn. To sum up, the main dynamic effects of the incomplete ionization are the delay of the device turn-off as the charge balance condition has to be restored in the device and the increase of the junction temperature. To tackle all these issues, the SJ diode unit cell could be redesigned.

\section{REDESIGN THE SUPERJUNCTION FOR IMPROVED DYNAMIC CHARGE BALANCE}

In the SJ theory the optimum breakdown voltage condition is met in the case of perfect balanced condition. This means that if the width and the doping of one of the two pillars are not fixed, it is possible to obtain a balanced SJ with an asymmetric device structure.
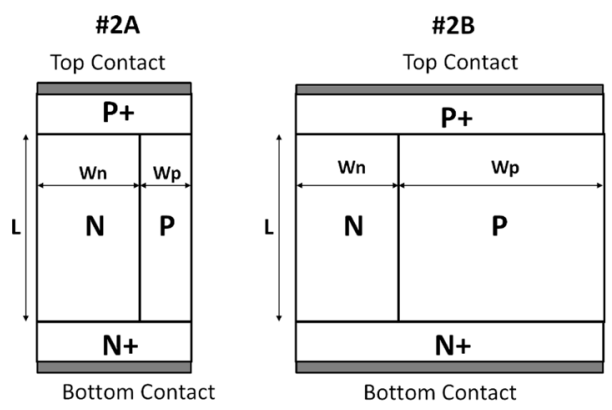

Figure 11, cross sections of the redesigned balanced SJ. The doping and the width of the n-type pillar have been fixed for all the different designs (as for the $\mathrm{S}-\mathrm{SJ}$ in figure 2) in order to perform a proper comparison of the performance. $\# 2 \mathrm{~A}$ has $\mathrm{N}_{\mathrm{A} 0}=1 \times 10^{17} \mathrm{~cm}^{-3}$ and $\mathrm{Wp}=0.5 \mu \mathrm{m}$ and $\# 2 \mathrm{~B}$ has $\mathrm{N}_{\mathrm{A} 0}=2.5 \times 10^{16} \mathrm{~cm}^{-3}$ and $\mathrm{Wp}=2 \mu \mathrm{m}$.

Figure 11 shows two different ways to redesign the SJ balance in order to tackle the dynamic unbalance. The charge balance condition is thus met for both structures and the static breakdown voltage is identical to the Symmetric SJ (S-SJ). The aspect ratio for all the different structures has been kept quite similar in order to maintain an identical breakdown voltage [36].

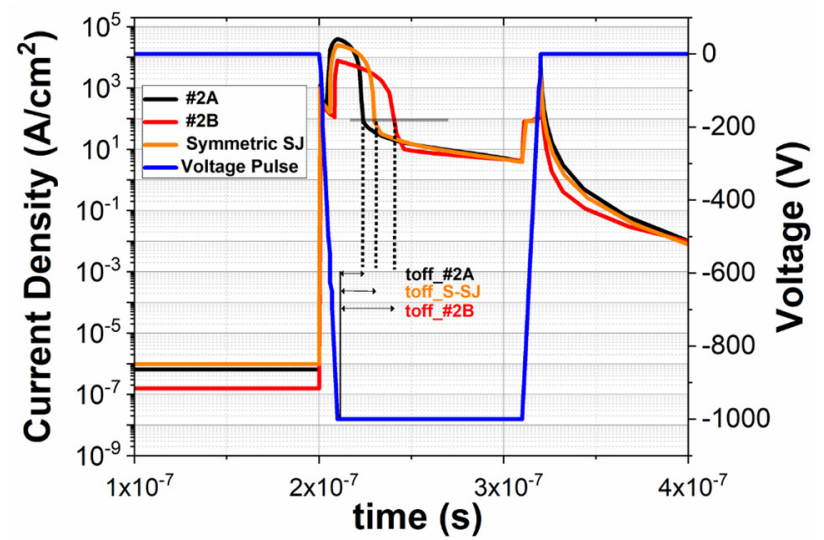

Figure 12, current density vs time for the 3 different SJ design. The turn-off time(toff), defined as the time where the current density reaches the value of $10^{2} \mathrm{~A} / \mathrm{cm}^{2}$, of $\# 2 \mathrm{~A}$ is much smaller than the other structures (toff $(\# 2 \mathrm{~A})=135 \mathrm{~ns}$, toff $(\# 2 B)=310 \mathrm{~ns}$, toff(S-SJ $=195 \mathrm{~ns})$. However, the dynamic current density peak obtained with \#2A is much higher.

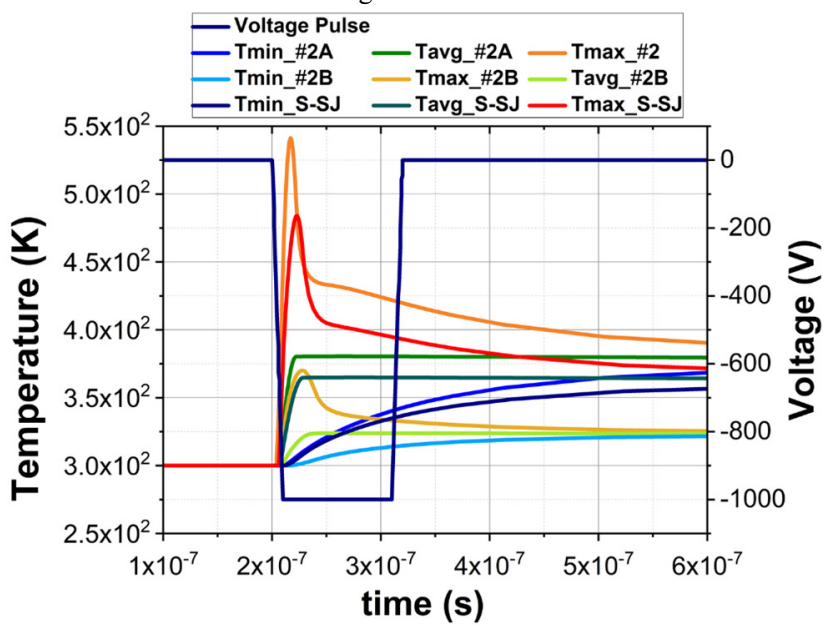

Figure 13, Temperature vs time for the 3 different SJ design. The maximum temperature is observed for $\# 2 \mathrm{~A}$ and it is $\sim 550 \mathrm{~K}$. The average temperatures for \#2A, \#2B and S-SJ are $375 \mathrm{~K}, 325 \mathrm{~K}, 360 \mathrm{~K}$, respectively. 
Figure 13 shows that the maximum, minimum and average temperature reached during the dynamic pulse is much higher for structure \#2A which has also a higher dynamic current density peak compared to the S-SJ and \#2B, as illustrated in figure 12. This happens because for higher doping concentration the unbalance ratio is much more significant, as it can be clearly deducted from figure 3(a). For this reason, the device operates in avalanche condition with a much higher unbalance ratio compared to the standard design and it heats up much faster as the avalanche current is also much higher. On the other hand, the enhanced self-heating is able to restore balance condition much faster than in the S-SJ and \#2B. Therefore, if the instantaneous junction temperature and current density increment can be considered as nondestructive mechanisms, it is possible to state that $\# 2 \mathrm{~A}$ reacts better to the dynamic unbalance with a reduction of the turn-off time defined as in figure 12. A fair performance comparison need to include also the on-state current density. The cross-sectional structures adopted for the on-state simulations are similar to figure 11, but an $\mathrm{n}+$ top layer (of $1 \mu \mathrm{m}$ ) has been inserted. The total current of structure \#2B will be much higher than the others as the depletion region mainly extends in the non-conductive pillar. However, the comparison need to be performed by inspecting the $\mathrm{R}_{\text {on_spec. }}$. The $\mathrm{R}_{\text {on_spec }}$ has been calculated in the linear region at room temperature (RT), as illustrated in the figure 14(a). \#2A has the lowest $R_{\text {on spec }}\left(0.34 \mathrm{~m} \Omega \cdot \mathrm{cm}^{2}\right)$ compared to $\# 1$ $\left(0.44 \mathrm{~m} \Omega \cdot \mathrm{cm}^{2}\right)$ and $\# 2 \mathrm{~B}\left(0.62 \mathrm{~m} \Omega \cdot \mathrm{cm}^{2}\right)$ at RT. In addition, also if $\mathrm{R}_{\text {on_spec }}$ is calculated at the average junction temperature reached by the device under transient condition (figure 13), \#2A outperforms the other device structures (figure $14 \mathrm{a}$ ). Distinct considerations need be done in case the incomplete ionization cannot be neglected in both pillars (as in diamond devices). In that specific circumstance, a more complex dynamic recovery takes place and the ionization time constants for both dopant species contribute to the rebalancing and the temperature distribution in the device.
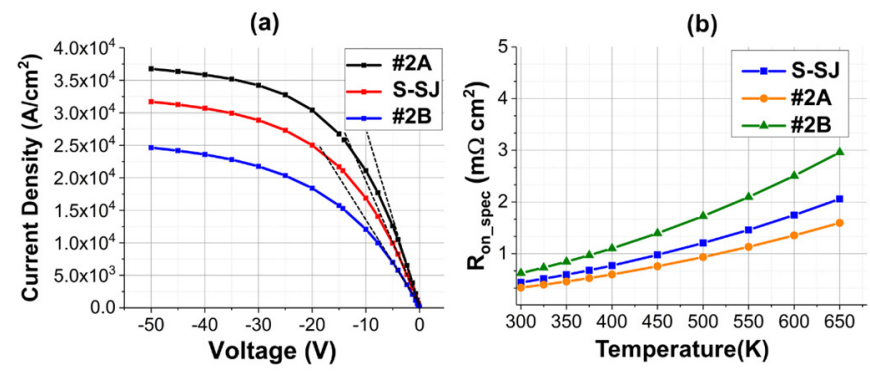

Figure 14, RT on-state current density for the $3 \mathrm{SJ}$ design (a) and specific onstate resistance vs Temperature (b).

\section{CONCLUSIONS}

The effect of the partial ionization of the dopants on the design of $4 \mathrm{H}-\mathrm{SiC}$ SJ diode has been analyzed in this paper. The static breakdown voltage is not influenced by the incomplete ionization effect as the high reverse electric field is able to activate all the dopants in the structure. However, when a very fast reverse pulse is applied to the SJ structure the dynamic ionization leads to an unbalance charge condition which has the effect of increasing the total current density and the local device temperature (up to $500 \mathrm{~K}$ in the example considered). It is nevertheless possible to redesign the $\mathrm{SJ}$ diode, while maintaining the ideal charge balance, in order to simultaneously improve the specific on-state resistance and the dynamic resistance to the charge imbalance due to incomplete ionization.

\section{REFERENCES}

[1] S. Shirota and S. Kaneda, "New type of varactor diode consisting of multilayer p-n junctions," Journal of Applied Physics, vol. 49, pp. 6012-6019, 1978. DOI: $10.1063 / 1.324570$

[2] D. J. Coe, "High voltage semiconductor devices, u.S. Patent ep0 053854 a1," Jun. 16, 1982.

[3] G. Deboy, M. Treu, O. Haeberlen, and D. Neumayr, "Si, sic and gan power devices: An unbiased view on key performance indicators," in Electron Devices Meeting (IEDM), 2016 IEEE International, 2016, pp. 20.2. 1-20.2. 4. DOI: $10.1109 /$ IEDM.2016.7838458

[4] T. P. Chow, I. Omura, M. Higashiwaki, H. Kawarada, and V. Pala, "Smart power devices and ics using gaas and wide and extreme bandgap semiconductors," IEEE Transactions on Electron Devices, vol. 64, pp. 856873, 2017. DOI: $10.1109 /$ TED.2017.2653759

[5] S. Chowdhury, Z. Guo, X. Liu, and T. P. Chow, "Comparison of silicon, sic and gan power transistor technologies with breakdown voltage rating from 1.2 kv to $15 \mathrm{kv}, "$ physica status solidi (c), vol. 13, pp. 354-359, 2016. . DOI: 10.1002/PSSC.201510200

[6] T. Fujihira, "Theory of semiconductor superjunction devices," Japanese journal of applied physics, vol. 36, p. 6254, 1997. DOI: 10.1143/JJAP.36.6254 [7] X. Chen, "Semiconductor power devices with alternating conductivity type high-voltage breakdown regions," ed: Google Patents, 1993.

[8] X. Chen, P. Mawby, K. Board, and C. Salama, "Theory of a novel voltagesustaining layer for power devices," Microelectronics journal, vol. 29, pp. 1005-1011, 1998. DOI: 10.1016/S0026-2692(98)00065-2

[9] E. Napoli, "Superjunction," Wiley Encyclopedia of Electrical and Electronics Engineering, 2014. DOI: 10.1002/047134608X.W8230

[10] F. Udrea, G. Deboy, and T. Fujihira, "Superjunction power devices, history, development, and future prospects," IEEE Transactions on Electron Devices, vol. 64, pp. 720-734, 2017. DOI: 10.1109/TED.2017.2658344

[11] S. Fujita, "Wide-bandgap semiconductor materials: For their full bloom," Japanese journal of applied physics, vol. 54, p. 030101, 2015. DOI: 10.7567/JJAP.54.030101

[12] A. Nakajima, Y. Sumida, M. H. Dhyani, H. Kawai, and E. Narayanan, "Gan-based super heterojunction field effect transistors using the polarization junction concept," IEEE Electron Device Letters, vol. 32, pp. 542-544, 2011. DOI: $10.1109 /$ LED.2011.2105242

[13] H. Ishida, D. Shibata, H. Matsuo, M. Yanagihara, Y. Uemoto, T. Ueda, T. Tanaka, and D. Ueda, "Gan-based natural super junction diodes with multichannel structures," in Electron Devices Meeting, 2008. IEDM 2008. IEEE International, 2008, pp. 1-4. DOI: 10.1109/IEDM.2008.4796636

[14] X. Zhong, B. Wang, J. Wang, and K. Sheng, "Experimental demonstration and analysis of a $1.35-\mathrm{kv} 0.92-\mathrm{m} \$ \omega \cdot \mathrm{cm}^{2}$ sic superjunction schottky diode," IEEE Transactions on Electron Devices, 2018. DOI: 10.1109/TED.2018.2809475

[15] R. Kosugi, Y. Sakuma, K. Kojima, S. Itoh, A. Nagata, T. Yatsuo, Y. Tanaka, and H. Okumura, "First experimental demonstration of sic superjunction (sj) structure by multi-epitaxial growth method," in Power Semiconductor Devices \& IC's (ISPSD), 2014 IEEE 26th International Symposium on, 2014, pp. 346-349. DOI: 10.1109/ISPSD.2014.6856047

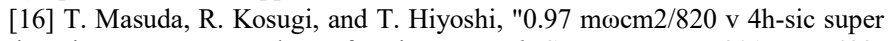
junction v-groove trench mosfet," in Materials Science Forum, 2017, pp. 483488. DOI: 10.4028/WwW.SCIENTIFIC.NET/MSF.897.483

[17] S. Koizumi, T. Teraji, and H. Kanda, "Phosphorus-doped chemical vapor deposition of diamond," Diamond and Related Materials, vol. 9, pp. 935-940, 2000. DOI: $10.1016 /$ S0925-9635(00)00217-X

[18] M. Lades, W. Kaindl, N. Kaminski, E. Niemann, and G. Wachutka, "Dynamics of incomplete ionized dopants and their impact on $4 \mathrm{~h} / 6 \mathrm{~h}$-sic 
devices," IEEE Transactions on Electron Devices, vol. 46, pp. 598-604, 1999. DOI: $10.1109 / 16.748884$

[19] "Synopsis manual, 2016"

[20] S. M. Sze and K. K. Ng. (2006). Physics of semiconductor devices. DOI: $10.1002 / 0470068329$

[21] N. Donato, D. Pagnano, E. Napoli, G. Longobardi, and F. Udrea, "Design of a normally-off diamond jet for high power integrated applications," Diamond and Related Materials, 2017. DOI: 10.1016/J.DIAMOND.2017.08.003 [22] G. Wellenhofer and U. Rössler, "Global band structure and near-band-edge states," physica status solidi (b), vol. 202, pp. 107-123, 1997. DOI: 10.1002/1521-3951

[23] G. Wachutka, "Consistent treatment of carrier emission and capture kinetics in electrothermal and energy transport models," Microelectronics journal, vol. 26, pp. 307-315, 1995. DOI: 10.1016/0026-2692(95)98933-I

[24] A. Schenk, "A model for the field and temperature dependence of shockley-read-hall lifetimes in silicon," Solid-State Electronics, vol. 35, pp. 1585-1596, 1992. DOI: 10.1016/0038-1101(92)90184-E

[25] W. Kaindl, M. Lades, N. Kaminski, E. Niemann, and G. Wachutka, "Experimental characterization and numerical simulation of the electrical properties of nitrogen, aluminum, and boron in $4 \mathrm{~h} / 6 \mathrm{~h}$-sic," Journal of electronic materials, vol. 28, pp. 154-160, 1999. DOI: 10.1007/s11664-999$\underline{0006-1}$

[26] A. V. Los and M. S. Mazzola, "Influence of carrier freeze-out on sic schottky junction admittance," Journal of electronic materials, vol. 30, pp. 235-241, 2001. DOI: 10.1007/s11664-001-0022-2

[27] H. Wang, E. Napoli, and F. Udrea, "Breakdown voltage for superjunction power devices with charge imbalance: An analytical model valid for both punch through and non punch through devices," IEEE Transactions on Electron Devices, vol. 56, pp. 3175-3183, 2009. DOI: 10.1109/TED.2009.2032595

[28] H. Niwa, J. Suda, and T. Kimoto, "Temperature dependence of impact ionization coefficients in 4h-sic," in Materials Science Forum, 2014, pp. 461466. DOI: $10.4028 /$ WWW.SCIENTIFIC.NET/MSF.778-780.461

[29] T. Hatakeyama, T. Watanabe, T. Shinohe, K. Kojima, K. Arai, and N. Sano, "Impact ionization coefficients of $4 \mathrm{~h}$ silicon carbide," Applied Physics Letters, vol. 85, pp. 1380-1382, 2004. DOI: $10.1063 / 1.1784520$

[30] C. Canali, G. Majni, R. Minder, and G. Ottaviani, "Electron and hole drift velocity measurements in silicon and their empirical relation to electric field and temperature," IEEE Transactions on Electron Devices, vol. 22, pp. 10451047, 1975. DOI: 10.1109/T-ED.1975.18267

[31] D. Disney and G. Dolny, "Jfet depletion in superjunction devices," in Power Semiconductor Devices and IC's, 2008. ISPSD'08. 20th International Symposium on, 2008, pp. 157-160. DOI: 10.1109/ISPSD.2008.4538922

[32] L. Efthymiou, G. Camuso, G. Longobardi, T. Chien, M. Chen, and F. Udrea, "On the source of oscillatory behaviour during switching of power enhancement mode gan hemts," Energies, vol. 10, p. 407, 2017. DOI: 10.3390/EN10030407

[33] Cree, " Z-rec® rectifier," vol. C3D02060E Silicon Carbide Schottky Diode, 2016.

[34] R. Semiconductor, "Sic power devices and modules - application note," Issue of August, 2014.

[35] G. K. Wachutka, "Rigorous thermodynamic treatment of heat generation and conduction in semiconductor device modeling," IEEE transactions on computer-aided design of integrated circuits and systems, vol. 9, pp. 11411149, 1990. DOI: $\underline{10.1109 / 43.62751}$

[36] L. Yu and K. Sheng, "Modeling and optimal device design for 4h-sic superjunction devices," IEEE Transactions on Electron Devices, vol. 55, pp. 19611969, 2008. DOI: 10.1109/TED.2008.926648 